\section{Studying lung cancer progression: insights from genetically engineered mouse models of cancer}

\author{
"Kras mutant mouse models of lung cancer suggest that Kras is a \\ promising target, as Kras withdrawal leads to tumor regression in \\ such models."
}

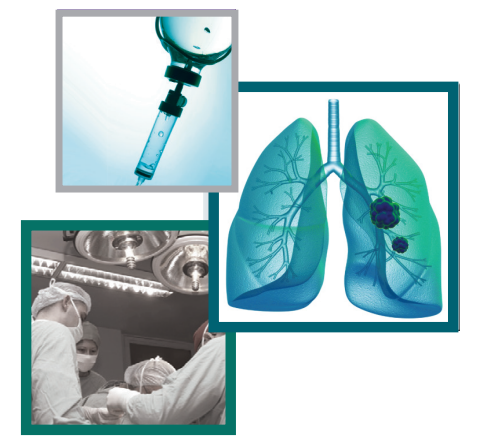

Sarah Neidler ${ }^{1} \&$ Daniel J Murphy ${ }^{*, 1}$

The most intuitive and in many cases successful way of targeting cancer is by inhibiting the driver mutation. This strategy improved treatment options for lung cancer patients with EGFR mutations [1], EML4-ALK fusion genes [2] and Ros1rearrangements [3]. However, a variety of mutations are found in adenocarcinomas of the lung and about a third of lung adenocarcinomas lack a known driver mutation. Another $30 \%$ of human NSCLC cases carry mutations in $K R A S$ [4] for which no targeted therapies are presently available. Kras is likely to be an initiating and driver mutation [5], but the mutational spectrum at the point of diagnosis is very heterogeneous and therefore very little is known about which subsequent alterations and mutations lead to disease progression.

A key breakthrough in lung cancer research came with the development of several mouse models with inducible ('conditional' in laboratory jargon) mutant Kras alleles cloned into the endogenous Kras locus [6,7]. Induction of the otherwise silent alleles is accomplished via acute, sporadic expression of a protein called CRE recombinase. The models thus allow for accurate control of the timing of disease initiation, enabling researchers to chart the entire course of disease progression over time. Crucially, upon sporadic activation in the lung these models faithfully recapitulate human NSCLC histopathologically as well as at the molecular level and mimic human lung cancer responses to standard therapies $[6,8]$. It is no overstatement to say that these models have revolutionized lung cancer research.

Kras mutant mouse models of lung cancer suggest that Kras is a promising target, as Kras withdrawal leads to tumor regression in such models [9]. Kras itself has proven difficult to target directly with small molecule inhibitors; however, several approaches to target Kras indirectly have emerged recently. One approach is to find synthetic lethal interactions - loss of gene functions that are lethal for Kras mutant cells but tolerated when Kras is wild-type. Another approach is to target downstream effectors such as MAPK and PI3K pathways. Both pathways are known to stabilize the oncogenic protein Myc [10].

Myc appears to be an obligate effector downstream of Kras as deletion of endogenous $M y c$ prevents Kras-driven tumorigenesis in multiple tissues [11,12]. As a transcription factor, Myc must heterodimerize with Max for binding to DNA and consequent regulation of transcription. A synthetic peptide, Omomyc, was developed to prevent Myc:Max heterodimerization and thereby block Myc function [13]. Expression of Omomyc in a Kras-driven mouse model of lung cancer lead to rapid tumor regression and progressive eradication, thereby establishing

IInstitute of Cancer Sciences, University of Glasgow, Glasgow, G12 8QQ, UK

*Author for correspondence: daniel.murphy@glasgow.ac.uk

\section{KEYWORDS}

- GEM models of lung cancer

- Kras $\bullet$ Myc $\bullet$ NSCLC $\bullet$ Wnt

“...30\% of human NSCLC cases carry mutations in KRAS, for which no targeted therapies are presently available." 
"Inducible mouse models of cancer facilitate the mechanistic analysis of tumor progression at early stages of disease development that are not readily accessible in the clinic, thereby yielding invaluable insights into pre-symptomatic disease.” the proof-of-principle of targeting Myc to treat cancer [13]. Another approach to targeting Myc, this time at the level of gene expression using small molecule inhibitors of bromodomain proteins, created a lot of excitement. Bromodomain containing proteins bind monoacetylated lysine residues that are found on $\mathrm{N}$-terminal tails of histones and one such protein, Brd4, regulates $M y c$ expression. The bromodomain and extra terminal bromodomain inhibitor JQ1 prevents this interaction and thereby suppresses $M y c$ expression [14]. This molecule has demonstrated remarkable anti-tumor activity in a Kras-driven model of NSCLC [15].

Interestingly, even though Kras signaling upregulates Myc by increasing its activity [10], Myc is additionally found to be amplified and mutated in $2-3 \%$ of Kras mutant human lung adenocarcinomas [16]. Moreover, MGA loss-offunction mutations, which increase Myc activity [4], are found in Kras mutant lung adenocarcinoma and are mutually exclusive with Myc amplification. Furthermore, in addition to being induced by upstream Kras signaling, Myc is also frequently deregulated via Krasindependent pathways, such as Wnt-pathway perturbation. The importance of Wnt signaling has recently been shown in a Braf-driven mouse model [17]. The frequency of Wnt pathway deregulation is difficult to accurately quantify; however, several canonical Wnt ligands are amplified in Kras-mutant lung adenocarcinoma [4]. This suggests that Myc levels may be limiting in Kras-driven tumorigenesis and thus that these tumors find additional ways to further upregulate Myc.

In the mouse, combining acute $\mathrm{Kras}^{\mathrm{G} 12 \mathrm{D}}$ induction with mild overexpression of c-Myc in the lung has been shown to accelerate Krasinduced tumor growth and to drive sporadic progression to adenocarcinoma, which is detectable as early as 6 weeks after allele induction [18].
Our current efforts are aimed at teasing apart the molecular basis of this progression with the dual goals of identifying candidate biomarkers of early stage lung cancer and new targets for therapeutic intervention.

These insights show that Kras is a promising target and that Myc plays a crucial role in Krasdriven tumorigenesis. But as both proteins are difficult to target directly, it also shows that a better understanding of lung adenocarcinoma progression will be needed in order to find additional candidate targets. Understanding the multistep process of tumor progression would yield important clues for future therapeutic intervention. Furthermore, identification of early tumor biomarkers (e.g., in blood) would enable detection of early-stage disease. At present, symptomatic patients typically present with advanced heterogenous disease that is difficult to treat effectively. Inducible mouse models of cancer facilitate the mechanistic analysis of tumor progression at early stages of disease development that are not readily accessible in the clinic, thereby yielding invaluable insights into presymptomatic disease. These insights will certainly give rise in time to new screening methods for early detection of lung and other cancers as well as revealing cancerspecific vulnerabilities for more effective and tumor-selective treatment of cancer.

\section{Financial \& competing interests disclosure}

$S$ Neidler is supported by an MINT collaborative grant from Merck sharpe \& Dohme pharmaceuticals to DJ Murphy and research relating to this article is further supported by the European Commision grant SERPLUC to DJ Murphy. The authors have no other relevant affiliations or financial involvement with any organization or entity with a financial interest in or financial conflict with the subject matter or materials discussed in the manuscript apart from those disclosed.

No writing assistance was utilized in the production of this manuscript.

\section{References}

1 Paez JG, Janne PA, Lee JC et al. EGFR mutations in lung cancer: correlation with clinical response to gefitinib therapy. Science 304(5676), 1497-1500 (2004).

2 Kwak EL, Bang YJ, Camidge DR et al. Anaplastic lymphoma kinase inhibition in non-small-cell lung cancer. N. Engl. J. Med. 363(18), 1693-1703 (2010).
3 Shaw AT, Ou SH, Bang YJ et al. Crizotinib in ROS1-rearranged non-small-cell lung cancer. N. Engl. J. Med. 371(21), 1963-1971 (2014).

4 Cancer Genome Atlas Research N. Comprehensive molecular profiling of lung adenocarcinoma. Nature 511(7511), 543-550 (2014).

5 Ding L, Getz G, Wheeler DA et al. Somatic mutations affect key pathways in lung adenocarcinoma. Nature 455(7216), 1069-1075 (2008).

6 Jackson EL, Willis N, Mercer K et al. Analysis of lung tumor initiation and progression using conditional expression of oncogenic K-RAS. Genes Dev. 15(24), 3243-3248 (2001).

7 Guerra C, Mijimolle N, Dhawahir A et al. Tumor induction by an endogenous $K$-RAS 
oncogene is highly dependent on cellular context. Cancer Cell 4(2), 111-120 (2003). Singh M, Murriel CL, Johnson L. Genetically engineered mouse models: closing the gap between preclinical data and trial outcomes. Cancer Res. 72(11), 2695-2700 (2012).

9 Fisher GH, Wellen SL, Klimstra D et al. Induction and apoptotic regression of lung adenocarcinomas by regulation of a $K-R A S$ transgene in the presence and absence of tumor suppressor genes. Genes Dev. 15(24), 3249-3262 (2001).

10 Sears R, Nuckolls F, Haura E, Taya Y, Tamai K, Nevins JR. Multiple RAS-dependent phosphorylation pathways regulate MYC protein stability. Genes Dev. 14(19), 2501-2514 (2000).
11 Walz S, Lorenzin F, Morton J et al. Activation and repression by oncogenic $M Y C$ shape tumour-specific gene expression profiles. Nature 511(7510), 483-487 (2014).

12 Oskarsson T, Essers MA, Dubois N et al. Skin epidermis lacking the $C-M Y C$ gene is resistant to $R A S$-driven tumorigenesis but can reacquire sensitivity upon additional loss of the P21CIP1 gene. Genes Dev. 20(15), 2024-2029 (2006).

13 Soucek L, Whitfield J, Martins CP et al. Modelling MYC inhibition as a cancer therapy. Nature 455(7213), 679-683 (2008).

14 Ott CJ, Kopp N, Bird L et al. BET bromodomain inhibition targets both C-MYC and IL7R in high-risk acute lymphoblastic leukemia. Blood 120(14), 2843-2852 (2012).
15 Shimamura T, Chen Z, Soucheray M et al. Efficacy of BET bromodomain inhibition in $K R A S$-mutant non-small cell lung cancer. Clin. Cancer Res. 19 (22), 6183-6192 (2013).

16 Gao J, Aksoy BA, Dogrusoz U et al. Integrative analysis of complex cancer genomics and clinical profiles using the cbioportal. Sci. Signal. 6(269), pl1 (2013).

17 Juan J, Muraguchi T, Iezza G, Sears RC, Mcmahon M. Diminished wnt -> betacatenin $\rightarrow$ C-MYC signaling is a barrier for malignant progression of $B R A F \mathrm{v} 600 \mathrm{e}-$ induced lung tumors. Genes Dev. 28(6), 561-575 (2014).

18 Murphy DJ, Junttila MR, Pouyet L et al. Distinct thresholds govern MYC's biological output in vivo. Cancer Cell 14(6), 447-457 (2008). 